\title{
Incidence Rate of Type 2 Diabetes Mellitus after Gestational Diabetes Mellitus: A Systematic Review and Meta-Analysis of 170,139 Women
}

\author{
Zhuyu Li, ${ }^{1}$ Yunjiu Cheng, ${ }^{2}$ Dongyu Wang, ${ }^{1}$ Haitian Chen, ${ }^{1}$ Hanqing Chen, ${ }^{1}$ Wai-kit Ming, ${ }^{1}$ \\ and Zilian Wang ${ }^{1}{ }^{1}$ \\ ${ }^{1}$ Department of Obstetrics and Gynaecology, The First Affiliated Hospital of Sun Yat-sen University, Guangzhou, China \\ ${ }^{2}$ Department of Cardiology, The First Affiliated Hospital of Sun Yat-sen University, Guangzhou, China \\ Correspondence should be addressed to Zilian Wang; wangzil@mail.sysu.edu.cn
}

Received 26 July 2019; Accepted 30 March 2020; Published 28 April 2020

Academic Editor: Maria Pia Francescato

Copyright $\odot 2020$ Zhuyu Li et al. This is an open access article distributed under the Creative Commons Attribution License, which permits unrestricted use, distribution, and reproduction in any medium, provided the original work is properly cited.

\begin{abstract}
Objective. The reported incidence of type 2 diabetes mellitus (T2DM) after gestational diabetes (GDM) varies widely. The purpose of this meta-analysis was to define the incidence rate of T2DM among women with a history of GDM and to examine what might modulate the rate. Research Design and Methods. We searched PubMed and Embase for terms related to T2DM after GDM up to January 2019. Large cohort studies with sample size $\geq 300$ and follow-up duration of at least one year were included. Data from selected studies were extracted, and meta-analysis was performed using the random-effects model. Subgroups analyses were based on the sample size of gestational diabetes, geographic region, maternal age, body-mass index, diagnostic criteria, and duration of follow-up. Results. Twenty-eight studies involving 170,139 women with GDM and 34,627 incident cases of T2DM were identified. The pooled incidence of T2DM after GDM was 26.20 (95\% CI, 23.31 to 29.10) per 1000 person-years. Women from Asia and those with older age and higher body mass index seem to experience higher risk of developing T2DM. The incidence rate of T2DM was lowest when applying IADPSG (7.16 per 1000 person-years) to diagnose GDM. The risk of developing T2DM after GDM increased linearly with the duration of follow-up. The increments per year of follow-up were estimated at $9.6 \%$. The estimated risks for T2DM were $19.72 \%$ at 10 years, $29.36 \%$ at 20 years, $39.00 \%$ at 30 years, $48.64 \%$ at 40 years, and $58.27 \%$ at 50 years, respectively. Conclusions. The findings of very high incidence of T2DM after GDM add an important insight into the trajectory of the development of T2DM in the long-term postpartum periods, which could provide evidence for consultant and might motivate more women with GDM to screen for T2DM. This trial is registered with PROSPERO identifier CRD42019128980.
\end{abstract}

\section{Introduction}

Gestational diabetes mellitus (GDM) is a condition in which glucose intolerance is first recognized during pregnancy. After delivery, these affected women are advised to perform glucose tolerance test as to screen for type 2 diabetes mellitus (T2DM) [1]. However, the low rate of postpartum screening for T2DM implies that we should make more efforts to improve their compliance [2]. Postpartum follow-up screening is often a responsibility for obstetricians who often pay more attention to pregnancy-related diseases but might ignore the conversion of GDM to T2DM. Although meta- analysis has shown that women with GDM have at least a seven-fold increased risk of developing T2DM, compared with those who have had a normoglycaemic pregnancy [3], there is no available meta-analysis on the incidence of T2DM after GDM. In addition, the reported incidence of T2DM after GDM varied widely from 1.3\% [4] to 70\% [5]. Consequently, demonstrating the incidence of T2DM among women with a history of GDM may help obstetricians attach more importance to this conversion and thus encourage more affected women to screen for T2DM.

Therefore, we conducted a meta-analysis with the following aims: (1) to ascertain the incidence rate of T2DM after 
GDM; (2) to perform a subgroup analysis based on study characteristics, including study designs, geographic region, sample sizes, age, body mass index (BMI), GDM, and T2DM criteria; and (3) to explore the link between duration of follow-up and incidence of T2DM after GDM.

\section{Materials and Methods}

2.1. Data Sources and Searches. This meta-analysis follows the MOOSE guidelines. We performed a comprehensive search of prospective, retrospective, randomized, or cohort studies in the electronic databases MEDLINE (source PubMed, January 1, 1966, to January 1, 2019) and Embase (January 1, 1980, to January 1, 2019) using the following text and key words in combination both as MeSH terms and text words "gestational diabetes," "diabetes mellitus," "type 2 diabetes mellitus," "NIDDM," and "non-insulin dependent diabetes mellitus." The search was limited to humans. We searched articles published in any language and scrutinized references from these studies to identify other relevant studies.

2.2. Study Selection. To be included in this meta-analysis, primary studies had to report the incidence rates of T2DM among the women with a history of GDM and the explicit follow-up duration. To enhance the representative and reliability, we made exclusion criteria as follows: (1) The amount of women with GDM in the study was less than 300. (2) The duration of follow-up was shorter than 12 months after the end of the index pregnancy. (3) There existed any form of intervention on preventing or delaying diabetes among women with gestational diabetes. (4) The study was a case series, letter, review, commentary, or editorial. For studies published in more than one report (duplicates), we considered the most comprehensive study that reported the largest sample size.

2.3. Data Extraction. Articles were reviewed and crosschecked independently by two authors (ZYL and YJC). The percentage agreement between the two authors on the quality review ranged from $89 \%$ to $100 \%$. Any disagreements were resolved by consensus between the authors (ZYL and YJC). The relevant data extracted included the year of publication, first author, source country, geographic region (defined as Europe, North America, Asia, etc.), study design, baseline patient characteristics, the total number of women with GDM and those with T2DM, follow-up data, and diagnostic criteria for GDM and T2DM.

2.4. Data Synthesis and Analysis. The primary end point of the study was the incidence rate of T2DM after GDM expressed as per 1000 person-years of follow-up and is presented with 95\% confidence intervals (CIs). The effect size was calculated using a random-effects model because we considered that the different patient groups in different countries during different periods were unlikely to have a common effect size. The mean duration of follow-up represented for the length of follow-up. Median length of follow-up could be used to estimate mean length because the sample size of each included study was larger than 25 according to the simulations derived from Hozo and colleagues [6].

Weighted meta-analytic prevalence estimates for outcomes were calculated with the variance-stabilizing Freeman-Tukey double-arcsine transformation with an inverse-variance random-effects model [7]. Heterogeneity was assessed with the $I^{2}$ statistic [8], where $I^{2}$ of at least $50 \%$ indicated significant heterogeneity. Sources of between-study heterogeneity were investigated using subgroup analysis.

We used a metaregression model to assess the relation between follow-up years and incidence rate of T2DM after GDM. We used Stata, version 14.0 (StataCorp) for all analyses. Statistical tests were two sided and used a significance level of $P<0.05$.

\section{Results}

3.1. Study Selection. With the search strategy, 1069 unique citations were initially retrieved. Of these, 159 articles were considered of interest and full text was retrieved for detailed evaluation. One hundred and thirty-one of these 159 articles were subsequently excluded, and finally, 28 articles were included in the meta-analysis (Figure 1).

3.2. Study Characteristics. Twenty-eight independent studies [9-36] reporting 170,139 women with GDM with 1,879,062 person-years of follow-up and a total of 34,627 incident cases of T2DM were identified (Table 1). The mean sample size of the studies was 6076 (range from 304 to 56,884). Fourteen studies $(n=155,340)$ were retrospective investigations, and the other fourteen studies $(n=14,799)$ were prospective.

The mean (SD) duration of follow-up was 8.35 (6.43) years (range, 1 to 30 years; interquartile range, 3.86 to 11.35 years). Patients were followed up for an average of over three years in a majority of studies (78.57\%).

Eleven studies were from Europe, seven from North America, six from Asia, two from Australasia, and two from intercontinental countries. Studies were published between January 1991 and September 2018.

3.3. Incidence of T2DM after GDM. All identified studies reported an incidence rate meeting all eligibility criteria for inclusion in a meta-analysis. The incidence proportion estimates ranged from 9.28 [16] per 1000 person-years to 96.10 [34] per 1000 person-years. The overall incidence rate of T2DM after GDM was 26.20 (95\% CI, 23.31 to 29.10 ) per 1000 person-years with very high between-sample heterogeneity $\left(P<0.001 ; I^{2}, 99.47 \%\right)$ (Figure 2$)$. The risk of developing T2DM after GDM increased linearly with the duration of follow-up by metaregression. The increment per year of follow-up was estimated to be $9.6 \%$ o (95\% CI, 3.6\%o$15.6 \%$ ). Accordingly, when the follow-up duration extended to 10 years, 20 years, 30 years, 40 years, and 50 years, the estimated risk of T2DM was $19.72 \%(7.44-32.01 \%), 29.36 \%$ (11.07-47.65\%), 39\% (14.71-63.29\%), 48.64\% (18.35$78.92 \%$ ), and $58.27 \%$ (21.99-94.56\%), respectively (Figure 3).

3.4. Subgroup Analysis. To explore the source of study heterogeneity, we performed stratified analyses across a number of key study characteristics and clinical factors, including 


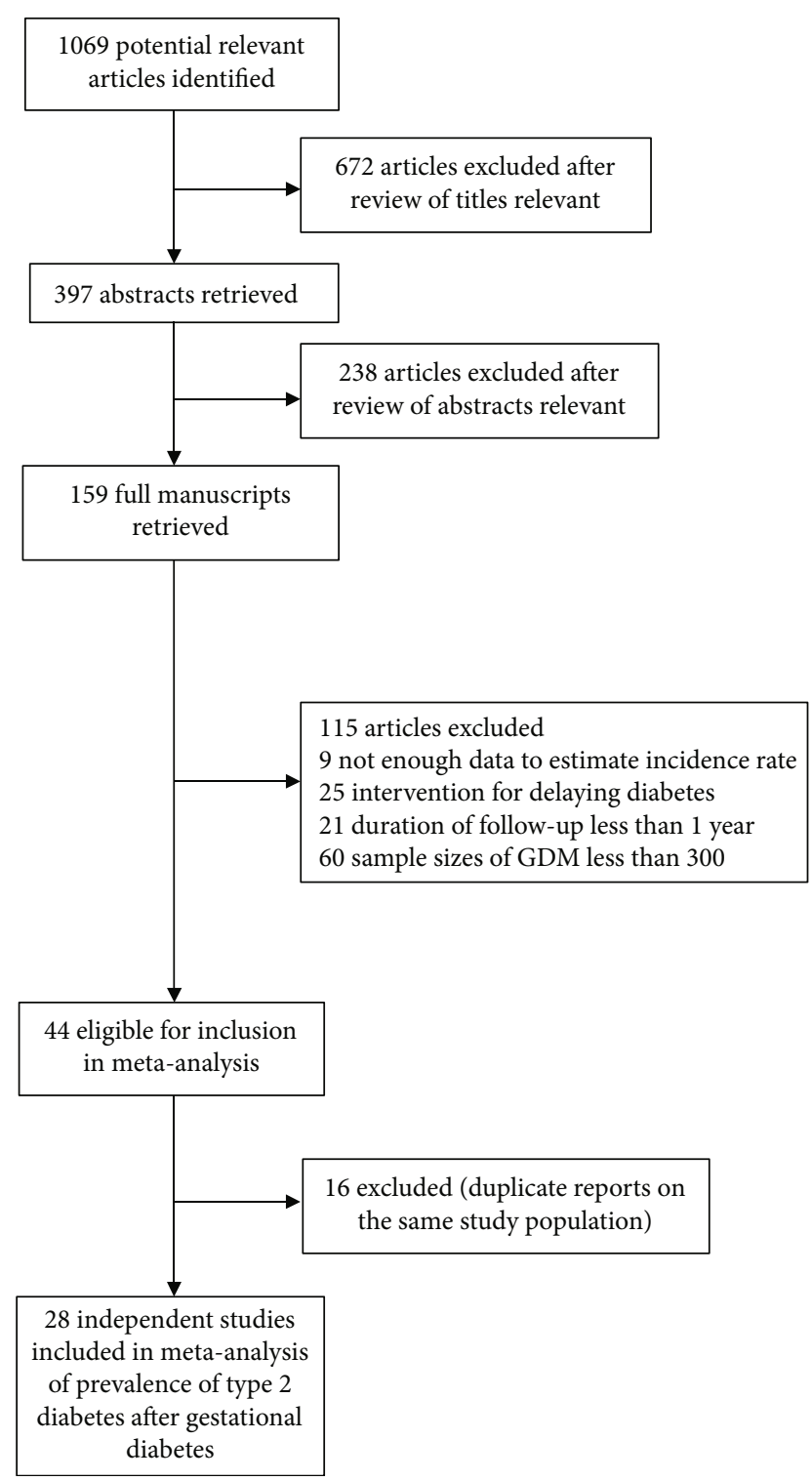

FIgURE 1: Flowchart of the selection of studies included in metaanalysis.

geographic region, baseline age, baseline BMI, study design, sample sizes, diagnostic criteria for GDM, and diagnostic criteria for T2DM (Figure 4).

Differences in T2DM rates after GDM according to geographic region were statistically significant $(P<0.001)$ (Figure S1). Women from Asia had the highest incidence rate of T2DM after GDM (45.96 per 1000 person-years), followed by those from North America (25.22 per 1000 person-years), Europe (25.17 per 1000 person-years), Australasia (18.12 per 1000 person-years), and intercontinental (12.51 per 1000 person-years).

Twenty-three studies of the identified studies demonstrated the baseline age. The mean (SD) baseline age was 30.8 (2.6) years old (range from 23.9 to 35.3). The incidence rate of T2DM after GDM was significantly higher among women with age $\geq 30$ years than those with age $<30$ years
(32.10 (95\% CI, 27.82-36.39) vs. 13.25 (95\% CI, $11.37-$ 15.12) per 1000 person-years, $P<0.001)$ (Figure S2).

Fifteen studies in our meta-analysis reported a baseline BMI. The mean (SD) baseline BMI was $25.7(2.9) \mathrm{kg} / \mathrm{m}^{2}$ (range from 20.9 to 30.9). As the average BMI of Asian women was lower than that of non-Asian women among the identified studies $\left(23.04 \mathrm{~kg} / \mathrm{m}^{2}\right.$ vs $\left.27.53 \mathrm{~kg} / \mathrm{m}^{2}\right)$, we conducted stratified analysis of baseline BMI by analyzing Asian and non-Asian women separately. Six studies from Asian countries and nine from non-Asian countries reported a baseline BMI. The incidence rate of T2DM after GDM was higher among Asian women whose $\mathrm{BMI} \geq 23 \mathrm{~kg} / \mathrm{m}^{2}$ (45.67 per 1000 person-years) than those with $\mathrm{BMI}<23 \mathrm{~kg} / \mathrm{m}^{2}$ (11.11 per 1000 person-years) $(P<0.001) \quad$ (Figure S3). Similarly, the incidence rate of T2DM after GDM was significantly higher in non-Asian women with baseline BMI $\geq 25 \mathrm{~kg} / \mathrm{m}^{2}$ than those with baseline BMI $<25 \mathrm{~kg} / \mathrm{m}^{2}$ $(22.08$ vs. 10.26 per 1000 person-years, $P<0.001)$ (Figure S4).

In addition, study design and sample size might influence the results. The incidence rate of T2DM of prospective studies was 31.79 per 1000 person-years, significantly higher than 22.68 per 1000 person-years of retrospective studies $(P=0.01)$ (Figure S5). Studies with small sample size seem to reported higher incidence rate than those with large sample size $(P<0.001)$ (Figure 56$)$.

Notably, the diagnostic criteria for GDM and T2DM in the primary studies also seem to be associated with the risk. The incidence of T2DM after GDM was highest when applying the Carpenter and Coustan (43.08) diagnostic criteria for GDM, followed by the International Workshop-Conference on GDM (39.23), NDDG (32.40), countrywide criteria (32.17), WHO (30.47), EASD (22.32), other (21.34), selfreported (11.06), and IADPSG (7.16 per 1000 person-years) $(P<0.001)$ (Figure S7). The incidence of T2DM after GDM for different diagnostic criteria for T2DM per 1000 personyears were as follows: 45.27 for ADA, 29.23 for NDDG, 28.13 for WHO, 22.94 for other criteria, 17.64 for mixed criteria, and 14.27 for self-reported $(P<0.001)$ (Figure $S 8)$.

Moreover, in one study by Cho [30], the incidence of T2DM stratified according to GDM status and the number of prepregnancy risk factors were reported. In women with or without GDM, the incidence rate of T2DM increased with the number of prepregnancy risk factors. Women with GDM had a higher incidence of T2DM than those without GDM but with the same number of prepregnancy risk factors. The incidence of T2DM was highest in women with both GDM and four prepregnancy risk factors (57.29 per 1000 person-years) and was lowest in those with neither GDM nor prepregnancy factors (3.19 per 1000 person-years) (Figure S9).

\section{Discussion}

The incidence rate of T2DM after GDM was found to be 26.20 per 1000 person-years through the present metaanalysis. The risk of developing T2DM was greater for women with GDM from Asia, with increased age, and with higher BMI in prospective studies, whereas the risk was lower 


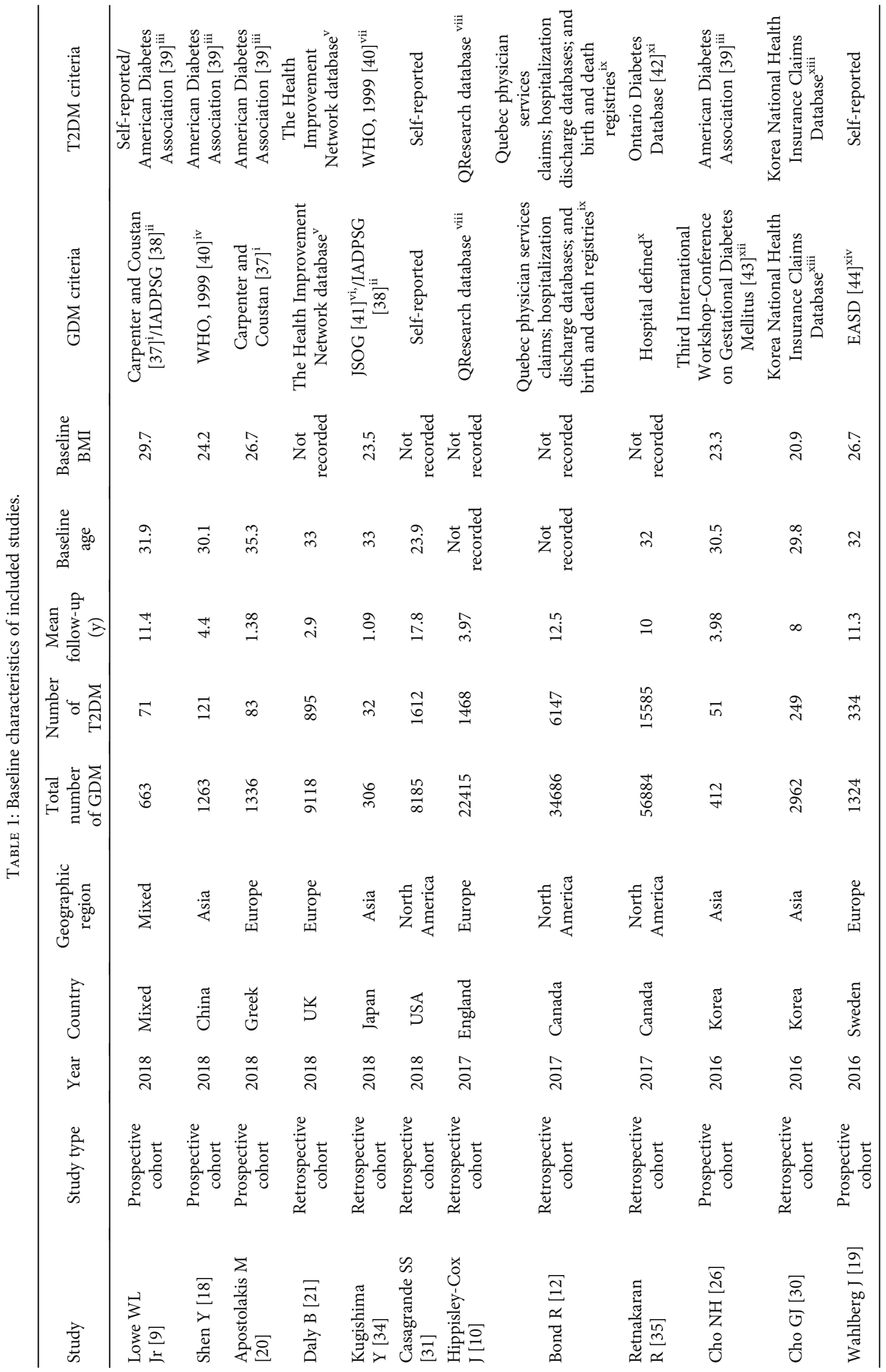




\begin{tabular}{|c|c|c|c|c|c|c|c|c|c|c|c|c|c|}
\hline 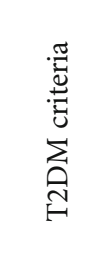 & 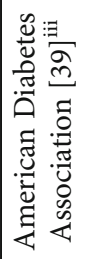 & 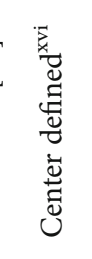 & 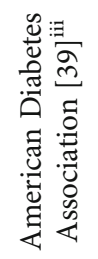 & 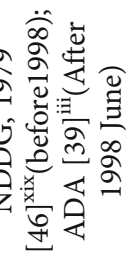 & 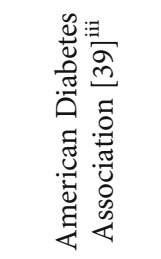 & 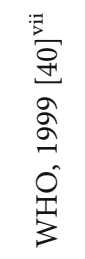 & 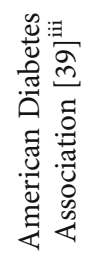 & 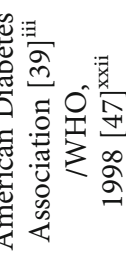 & $\begin{array}{l}\text { 串 } \\
\stackrel{0}{0}\end{array}$ & $\begin{array}{l}3 \\
0 \\
0 \\
3 \\
3\end{array}$ & 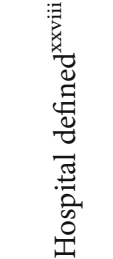 & 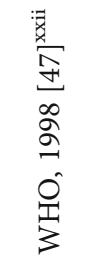 & 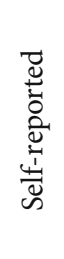 \\
\hline 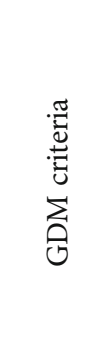 & 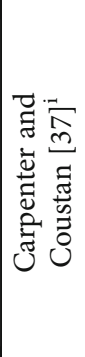 & 仓 & 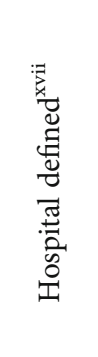 & 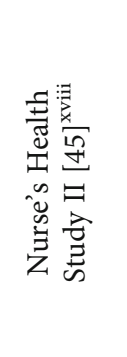 & 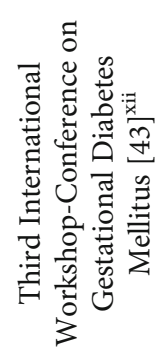 & 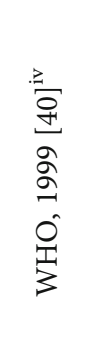 & 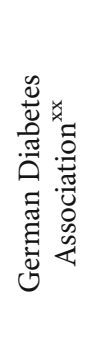 & 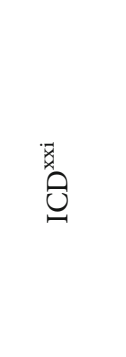 & 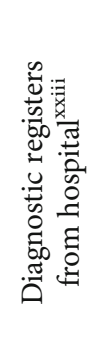 & 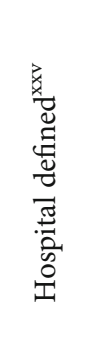 & 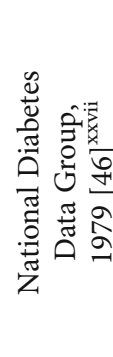 & 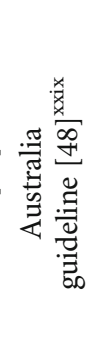 & \\
\hline 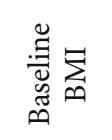 & in & 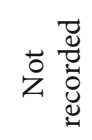 & @े & $\widehat{\curvearrowright}$ & $\begin{array}{l}\infty \\
\stackrel{\sim}{i}\end{array}$ & z & 艺䔅 & Z & 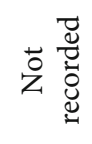 & ż & $\stackrel{\stackrel{1}{n}}{\sim}$ & જે & \\
\hline 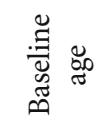 & $\ddot{m}$ & $\begin{array}{l}\infty \\
\infty \\
\infty \\
\sim\end{array}$ & $\tilde{m}$ & 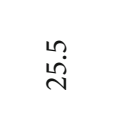 & $\underset{m}{\stackrel{m}{m}}$ & 范 & $\vec{m}$ & $\stackrel{\infty}{\stackrel{\infty}{0}}$ & 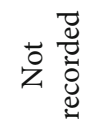 & 雚 & $\underset{m}{\stackrel{m}{m}}$ & $\hat{\text { si }}$ & $\underset{m}{\stackrel{0}{m}}$ \\
\hline 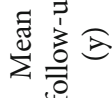 & $\stackrel{+}{\stackrel{ \pm}{-}}$ & ి & $\stackrel{-}{ }$ & $\stackrel{n}{2}$ & 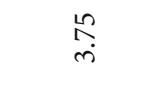 & $\stackrel{\stackrel{n}{ }}{\stackrel{f}{+}}$ & $\stackrel{\leftrightarrow}{\curvearrowright}$ & $\underset{\infty}{b}$ & $\exists$ & $\neg$ & $\vec{i}$ & $\stackrel{10}{\sim}$ & $\hat{\imath}$ \\
\hline 离 & $\cong$ & ڤ̊ & $\vec{n}$ & $\hat{n}$ & $\stackrel{\varrho}{\exists}$ & $\stackrel{\rightleftarrows}{=}$ & 丹 & స్ల & $\stackrel{\infty}{\sim}$ & $\stackrel{\infty}{m}$ & $\vec{\nabla}$ & $\vec{\exists}$ & $\ddot{\sim}$ \\
\hline 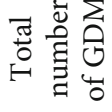 & ڤू & 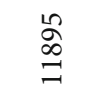 & $\stackrel{\partial}{a}$ & $\begin{array}{l}\text { 芯 } \\
\stackrel{2}{6}\end{array}$ & 足 & হ̊ & 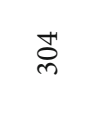 & $\underset{\exists}{\exists}$ & iิ & 앙 & ర్రి & 尽 & $\stackrel{\mathscr{p}}{\not}$ \\
\hline 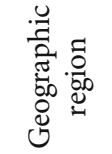 & 吾泀 & 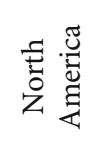 & 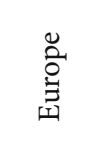 & 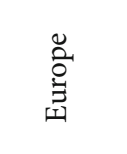 & $\frac{\pi}{4}$ & 荧 & $\begin{array}{l}\text { Ŏ. } \\
\stackrel{\Xi}{\Xi} \\
\text { ज्ञ }\end{array}$ & 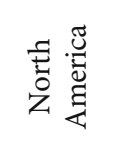 & 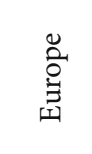 & 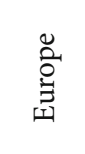 & $\frac{\pi}{\frac{\pi}{4}}$ & 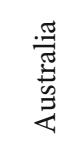 & 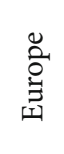 \\
\hline 吾 & 岕 & 䒕 & $\begin{array}{l}\overrightarrow{\widetilde{E}} \\
\text { 遥 }\end{array}$ & 光 & 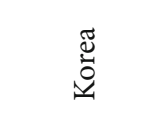 & 离 & 气ี & 崩 & 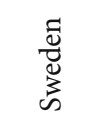 & 光 & $\begin{array}{l}\mathscr{J} \\
\stackrel{\tilde{\sigma}}{v}\end{array}$ & 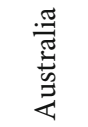 & 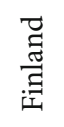 \\
\hline 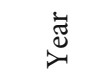 & $\stackrel{n}{\stackrel{n}{*}}$ & $\stackrel{n}{\stackrel{2}{*}}$ & $\stackrel{n}{\stackrel{1}{*}}$ & 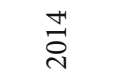 & $\stackrel{n}{\vec{N}}$ & 퐁 & $\stackrel{\widetilde{\pi}}{\tilde{N}}$ & 곡 & 곡 & $\stackrel{\circ}{\stackrel{1}{*}}$ & 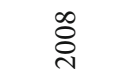 & ث્ণે & ఫ্ণ \\
\hline 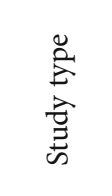 & 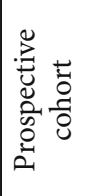 & 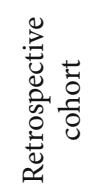 & 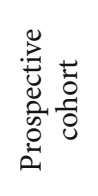 & 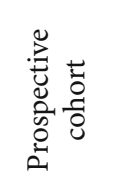 & 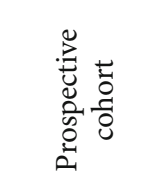 & 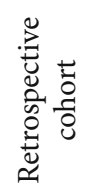 & 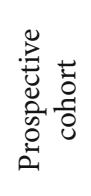 & 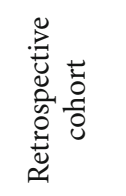 & 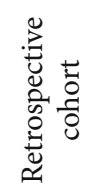 & 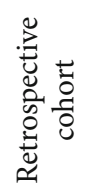 & 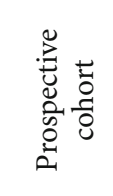 & 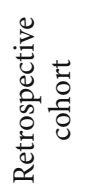 & \\
\hline 苞 & 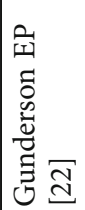 & 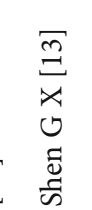 & 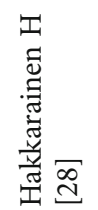 & $\begin{array}{l}\sqrt{\mathrm{N}} \\
3 \\
0 \\
0 \\
\infty\end{array}$ & 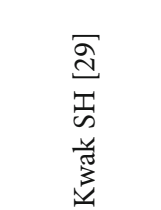 & 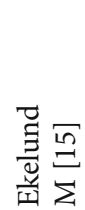 & 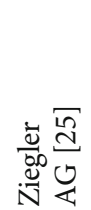 & 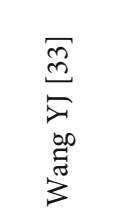 & 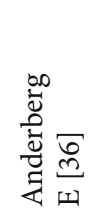 & 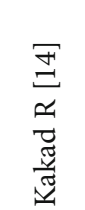 & 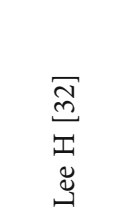 & 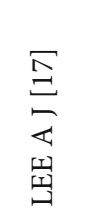 & 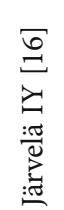 \\
\hline
\end{tabular}




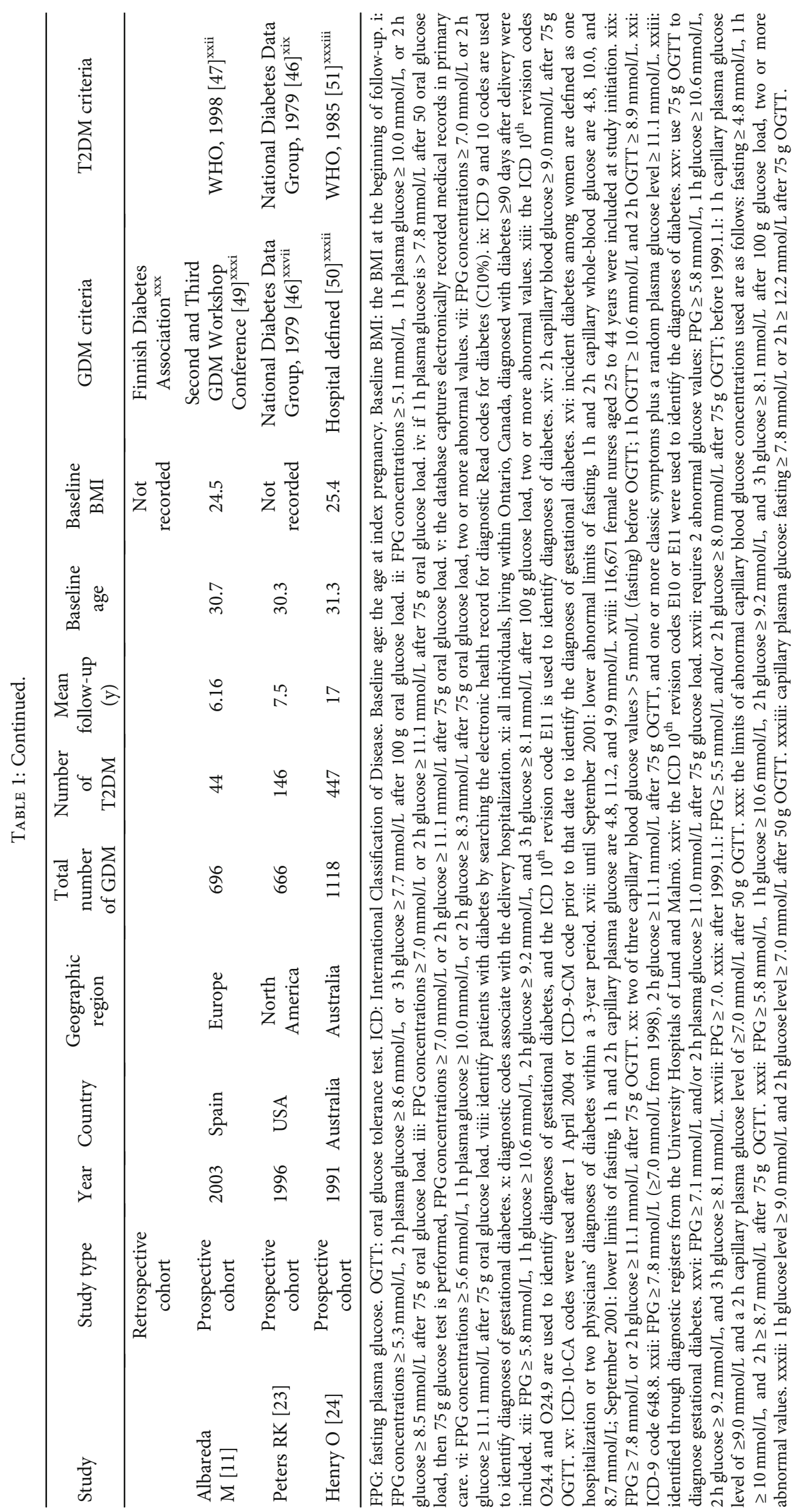




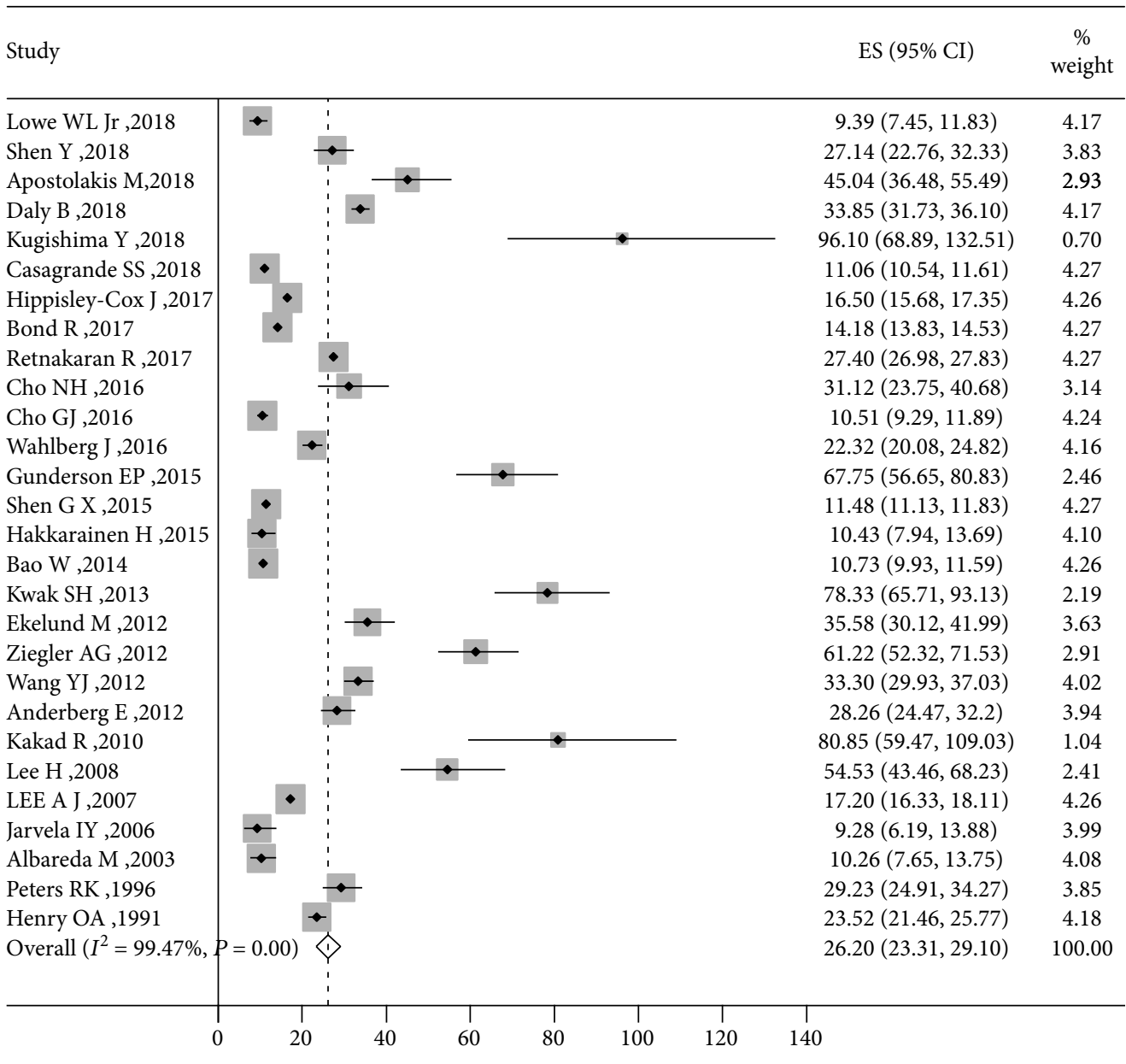

Figure 2

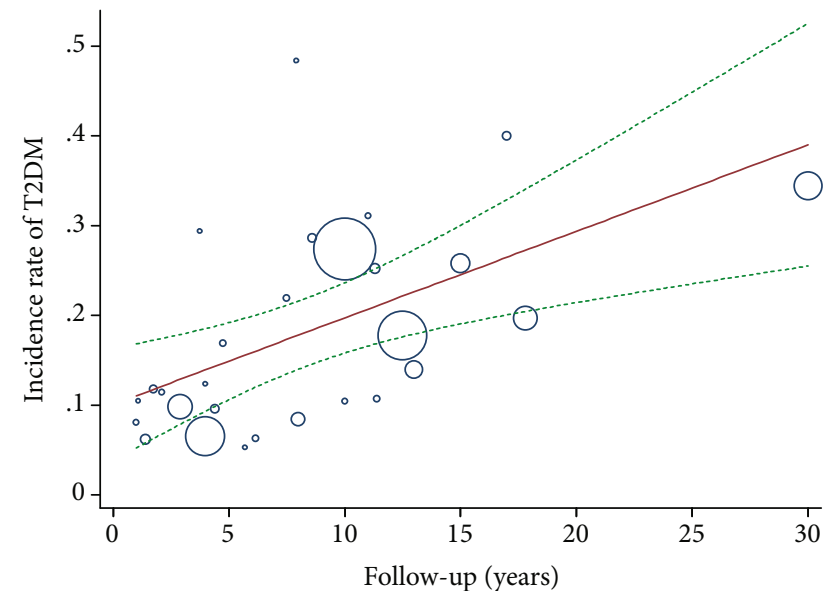

FIGURE 3

when applying the IADPSG criteria to diagnose GDM. Furthermore, the risk of developing T2DM increased linearly with the duration of follow-up.

Although quite a few reviews and meta-analysis [3, 52-55] have evaluated the relative risk of T2DM after GDM, there are no available meta-analyses on the incidence rate of T2DM after GDM. This meta-analysis is the first to our knowledge to calculate the incidence rate of T2DM among women with a history of GDM.

The incidence rates of T2DM quoted in our included literatures have been extremely variable. Some characters of the studies affect the estimate rate. The highest conversion from GDM to T2DM in Asian populations in our finding might, in part, explain why Asia has emerged as the major area with a rapidly developing T2DM epidemic [56]; apart from that, Asia has the highest prevalence of GDM [57]. In Asian countries, the T2DM epidemic is characterized by onset at a lower BMI than in Western populations [58], which is also consistent with our finding. That is also why we performed subgroup analysis of baseline BMI by separating Asian and non-Asian populations and according to Asia-specific BMI cut points [59] in Asian populations. However, the reasons why Asians have a higher risk of T2DM at a lower BMI are unclear. It has been suggested that for a given BMI, Asians have a higher percentage of body fat and more visceral adipose tissue compared with other racial/ethnic groups [60]. Low BMI and a tendency toward greater abdominal obesity put Asian people at high risk of $\beta$-cell dysfunction and insulin resistance thus triggering T2DM [58].

Aside from populations, the large variation in the subsequent development of type 2 diabetes may also be due to the 


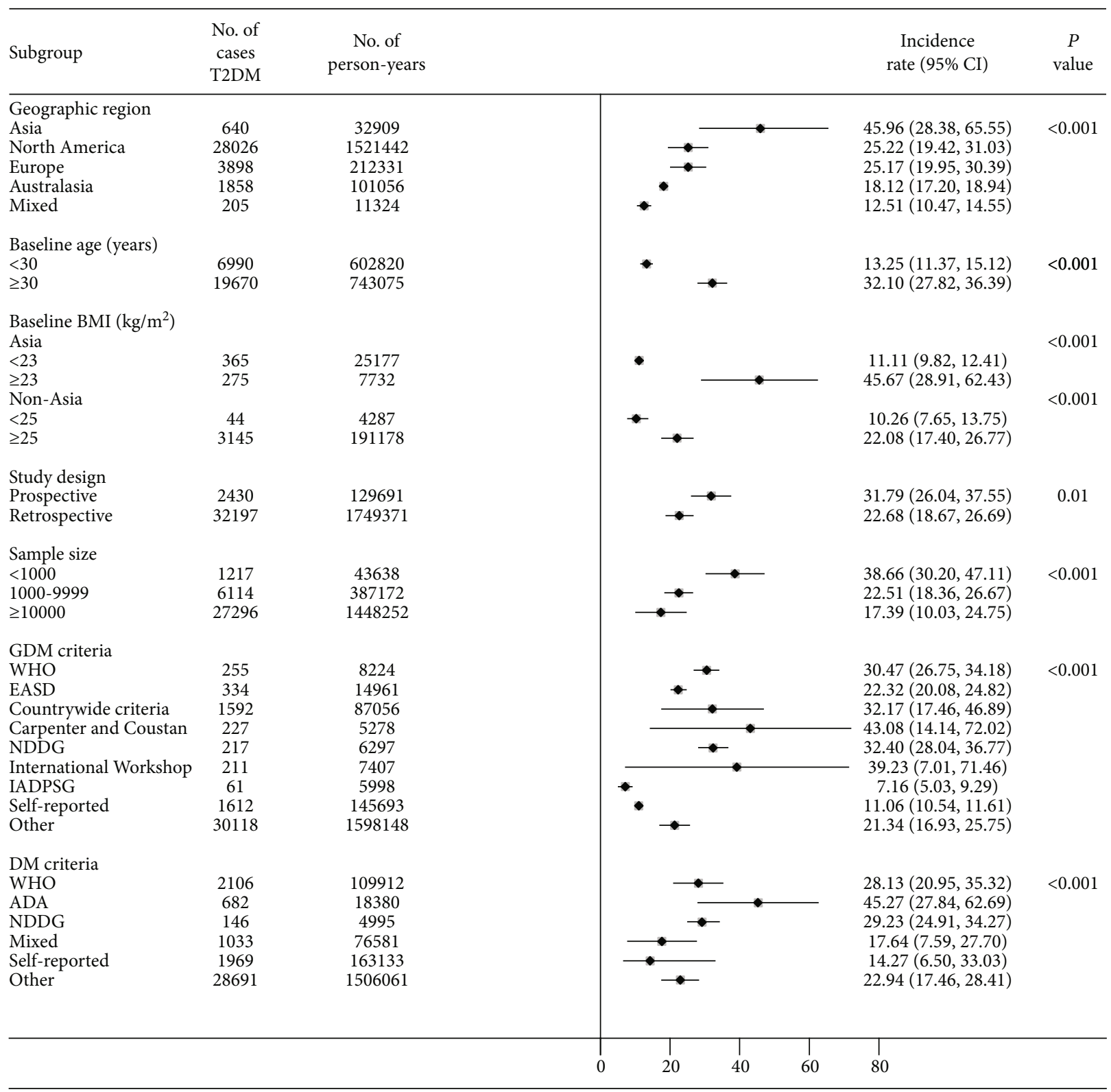

FiguRE 4

use of diverse diagnostic criteria for GDM. Diagnostic criteria for GDM have rapidly evolved during the past few decades. The IADPSG recommendations [38] are the first evidence-based, large-scale guideline established in 2010 based on glucose levels associated with adverse pregnancy outcomes in the HAPO Study [61] and in other studies. Duran et al. found the application of the new IADPSG criteria was associated with a 3.5-fold increase in GDM prevalence compared with the traditional Carpenter-Coustan criteria [62], as well as significant improvements in pregnancy outcomes. But there are few studies exploring the long-term outcomes of IADPSG-defined GDM until now. We found the incidence rate of T2DM was lowest among those with IADPSG-defined GDM (7.16 per 1000 personyears) compared with GDM defined by other diagnostic criteria. In the ATLANTIC-DIP study of white Europeans [63],
270 women with a history of GDM based on IADPSG criteria were followed up to 5 years (a mean follow-up of 2.6 years) postpartum. In total, six women demonstrated T2DM; thus, the incidence rate of 8.55 per 1000 person-years was similar to our finding. They also found the that cumulative incidence of abnormal glucose tolerance between women meeting the IADPSG criteria only and those meeting the modified WHO 1999 criteria only was of no significant differences $(P=0.798)$. It seems their findings were different from ours. However, it is uncertain, since they assessed incidence of abnormal glucose tolerance which included impaired fasting glucose, impaired glucose tolerance, and diabetes. Even if the incidence rate of T2DM after the IADPSG-defined GDM reduced, given the rising prevalence of GDM defined by IADPSG, the trend of T2DM associated with GDM is unclear which needs to be explored further. 
Notably, the risk of developing T2DM after GDM tended to increase linearly with the duration of follow-up. For example, women who suffered from GDM during the age of 31 years old would have a risk of $19.72 \%$ for developing T2DM in the age 41 years old and a risk of $39 \%$ in the age 61 years old. A systematic review including 28 studies performed by Kim et al. in 2002 [53] showed that the progression to type 2 diabetes increased markedly within the first 5 years after delivery and appeared to plateau after ten years, which was not consistent with ours. The substantial differences might lie in the duration of follow-up. The follow-up time was shorter than five years among $67.86 \%$ of his studies, while $60.71 \%$ of our studies had a duration of follow-up of more than five years after GDM.

Our study has important implications. Existing reviews often accessed the relative risk of T2DM among GDM and their peers, which could not give an intuitive concept to physicians and patients. Our study provided an absolute risk of T2DM among women with GDM, which might motivate these affected mothers to attend screening programmes thus help guide lifestyle management and monitoring to reduce the future risk of T2DM.

Strengths of this meta-analysis include the strict inclusion criteria, the large number of patients analyzed, the robustness of the findings in subgroup analyses, and the relationship between follow-up duration and risk of T2DM.

Like all meta-analyses, our study has the limitation of being a retrospective analysis. Another limitation is the lack of individual participant data, which precluded stratifying results according to insulin use during pregnancy, family history of diabetes, and polycystic ovary syndrome which are the high risk factors for T2DM. However, in the study by Cho, the incidence rate of T2DM seemed to be associated with the number of prepregnancy risk factors. It suggests that multiple prepregnancy risk factors other than GDM might also increase the risk of developing T2DM. Further studies, including well-designed clinical trials, are warranted to elucidate the specific pathogenic mechanisms and the impact of other potential risk factors.

Despite these limitations, this study is, to our knowledge, the first meta-analysis to quantify the future onset risk of T2DM in women with GDM. The findings add an important insight into the trajectory of the development of type 2 diabetes in the long-term postpartum periods.

\section{Conclusion}

In conclusion, this meta-analysis provided overall estimates of T2DM incidence rates in women with previous GDM, which showed substantial differences according to geographic region, age, baseline BMI, and diagnostic criteria for GDM and T2DM. Furthermore, we found that the risk of T2DM increased linearly by $9.6 \%$ for every additional one year of follow-up after GDM.

\section{Data Availability}

The data used to support the findings of this study are available from corresponding author upon request.

\section{Disclosure}

All authors have no conflicts of interest to disclose.

\section{Conflicts of Interest}

The authors declares that they have no conflicts of interest.

\section{Authors' Contributions}

ZYL and YJC contributed equally and were joint first authors. ZLW, ZYL, and YJC conceived the study concept and design. ZYL, YJC, DYW, and HTC contributed to data extraction. ZYL, YJC, HQC, WKM, and ZLW contributed to statistical analysis. All authors contributed to data interpretation. ZYL, YJC, and ZLW contributed to the writing of the manuscript, and all authors provided critical revisions for important intellectual content. Zhuyu $\mathrm{Li}$ and Yunjiu Cheng contributed equally to this work.

\section{Acknowledgments}

This work is funded by the 5010 Project of Sun Yat-sen University (No. 2012004) and National Natural Science Foundation of China (No. 81571452).

\section{Supplementary Materials}

Figure S1: forest plot of incidence rates of T2DM after GDM per 1000 person-years of follow-up stratified by geographic region. Horizontal lines indicate 95\% CIs. Differences in T2DM rates after GDM according to geographic region were statistically significant $(P<0.001)$. Figure S2: forest plot of incidence rates of T2DM after GDM per 1000 person-years of follow-up stratified by baseline age. Horizontal lines indicate $95 \%$ CIs. The incidence rate of T2DM after GDM was significantly higher among women with age $\geq 30$ years than those with age $<30$ years $(P<0.001)$. Figure S3: forest plot of incidence rates of T2DM after GDM per 1000 personyears of follow-up stratified by BMI of Asian. Horizontal lines indicate $95 \%$ CIs. The incidence rate of T2DM after GDM was higher among Asian women whose BMI $\geq 23 \mathrm{~kg} /$ $\mathrm{m}^{2}$ than those with BMI $<23 \mathrm{~kg} / \mathrm{m}^{2}(P<0.001)$. Figure S4: forest plot of incidence rates of T2DM after GDM per 1000 person-years of follow-up stratified by BMI of non-Asian. Horizontal lines indicate 95\% CIs. The incidence rate of T2DM after GDM was significantly higher in non-Asian women with baseline BMI $\geq 25 \mathrm{~kg} / \mathrm{m}^{2}$ than those with baseline $\mathrm{BMI}<25 \mathrm{~kg} / \mathrm{m}^{2} \quad(P<0.001)$. Figure S5: forest plot of incidence rates of T2DM after GDM per 1000 person-years of follow-up stratified by study design. Horizontal lines indicate 95\% CIs. The incidence rate of T2DM of prospective studies was significantly higher than retrospective studies $(P=0.01)$. Figure $S 6$ : forest plot of incidence rates of T2DM after GDM per 1000 person-years of follow-up stratified by sample size of GDM. Horizontal lines indicate $95 \%$ CIs. Studies with small sample size reported higher incidence rate than those with large sample size $(P=0.01)$. Figure S7: forest plot of incidence rates of T2DM after GDM per 1000 person-years of follow-up stratified by GDM criteria. 
Horizontal lines indicate 95\% CIs. The incidence of T2DM after GDM was highest when applying the Carpenter and Coustan (43.08) diagnostic criteria for GDM and was lowest when applying IADPSG (7.16 per 1000 person-years) $(P<0.001)$. Figure S8: forest plot of incidence rates of T2DM after GDM per 1000 person-years of follow-up stratified by T2DM criteria. Horizontal lines indicate 95\% CIs. The incidence of T2DM after GDM for different diagnostic criteria for T2DM per 1000 person-years was significantly different $(P<0.001)$. Figure S9: incidence rate of type 2 diabetes stratified by GDM status and the number of prepregnancy risk factors according to the study of Cho [30]. (Supplementary Materials)

\section{References}

[1] American Diabetes Association, "Standards of medical care in Diabetes-2019Abridged for primary care providers," Clinical Diabetes, vol. 37, no. 1, pp. 11-34, 2019.

[2] O. Adekojo, K. R. Revell, H. Preece, S. Morris, M. A. Coleman, and R. I. G. Holt, "Low uptake of postpartum screening for type 2 diabetes in women after a diagnosis of gestational diabetes," Diabetic Medicine, vol. 33, no. 11, pp. 1599-1601, 2016.

[3] L. Bellamy, J. P. Casas, A. D. Hingorani, and D. Williams, "Type 2 diabetes mellitus after gestational diabetes: a systematic review and meta-analysis," The Lancet, vol. 373, no. 9677, pp. 1773-1779, 2009.

[4] J. Ogonowski and T. Miazgowski, "The prevalence of 6 weeks postpartum abnormal glucose tolerance in Caucasian women with gestational diabetes," Diabetes Research and Clinical Practice, vol. 84, no. 3, pp. 239-244, 2009.

[5] N. Mohamed and J. Dooley, "Gestational diabetes and subsequent development of NIDDM in aboriginal women of northwestern Ontario," International Journal of Circumpolar Health, vol. 57, Supplement 1, pp. 355-358, 1998.

[6] S. P. Hozo, B. Djulbegovic, and I. Hozo, "Estimating the mean and variance from the median, range, and the size of a sample," BMC Medical Research Methodology, vol. 5, no. 1, p. 13, 2005.

[7] J. J. Miller, "The inverse of the Freeman - Tukey double arcsine transformation," The American Statistician, vol. 32, no. 4, p. 138, 1978.

[8] R. J. Hardy and S. G. Thompson, "Detecting and describing heterogeneity in meta-analysis," STAT MED, vol. 17, no. 8, pp. 841-856, 1998.

[9] W. J. Lowe Jr., D. M. Scholtens, L. P. Lowe et al., “Association of gestational diabetes with maternal disorders of glucose metabolism and childhood adiposity," JAMA, vol. 320, no. 10, pp. 1005-1016, 2018.

[10] J. Hippisley-Cox and C. Coupland, "Development and validation of QDiabetes-2018 risk prediction algorithm to estimate future risk of type 2 diabetes: cohort study," $B M J$, vol. 359, article j5019, 2017.

[11] M. Albareda, A. Caballero, G. Badell et al., "Diabetes and abnormal glucose tolerance in women with previous gestational diabetes," Diabetes Care, vol. 26, no. 4, pp. 1199-1205, 2003.

[12] R. Bond, R. Pace, E. Rahme, and K. Dasgupta, "Diabetes risk in women with gestational diabetes mellitus and a history of polycystic ovary syndrome: a retrospective cohort study," Diabetic Medicine, vol. 34, no. 12, pp. 1684-1695, 2017.
[13] G. X. Shen, L. A. Shafer, P. J. Martens et al., "Does First Nations ancestry modify the association between gestational diabetes and subsequent diabetes: a historical prospective cohort study among women in Manitoba, Canada," Diabetic Medicine, vol. 33, no. 9, pp. 1245-1252, 2015.

[14] R. Kakad, A. Anwar, P. Dyer, J. Webber, and J. Dale, "Fasting plasma glucose is not sufficient to detect ongoing glucose intolerance after pregnancy complicated by gestational diabetes," Experimental and Clinical Endocrinology \& Diabetes, vol. 118, no. 4, pp. 234-236, 2010.

[15] M. Ekelund, N. Shaat, P. Almgren et al., "Genetic prediction of postpartum diabetes in women with gestational diabetes mellitus," Diabetes Research and Clinical Practice, vol. 97, no. 3, pp. 394-398, 2012.

[16] I. Y. Jarvela, J. Juutinen, P. Koskela et al., "Gestational diabetes identifies women at risk for permanent type 1 and type 2 diabetes in fertile age: predictive role of autoantibodies," Diabetes Care, vol. 29, no. 3, pp. 607-612, 2006.

[17] A. J. Lee, R. J. Hiscock, P. Wein, S. P. Walker, and M. Permezel, "Gestational diabetes mellitus: clinical predictors and longterm risk of developing type 2 diabetes: a retrospective cohort study using survival analysis," Diabetes Care, vol. 30, no. 4, pp. 878-883, 2007.

[18] Y. Shen, P. Wang, L. Wang et al., "Gestational diabetes with diabetes and prediabetes risks: a large observational study," European Journal of Endocrinology, vol. 179, no. 1, pp. 5158, 2018.

[19] J. Wahlberg, B. Ekman, L. Nyström, U. Hanson, B. Persson, and H. J. Arnqvist, "Gestational diabetes: glycaemic predictors for fetal macrosomia and maternal risk of future diabetes," Diabetes Research and Clinical Practice, vol. 114, pp. 99-105, 2016.

[20] M. Apostolakis, S. A. Paschou, E. Zapanti, V. Sarantopoulou, V. Vasileiou, and E. Anastasiou, "HbA1c presents low sensitivity as a post-pregnancy screening test for both diabetes and prediabetes in Greek women with history of gestational diabetes mellitus," Hormones, vol. 17, no. 2, pp. 255-259, 2018.

[21] B. Daly, K. A. Toulis, N. Thomas et al., "Increased risk of ischemic heart disease, hypertension, and type 2 diabetes in women with previous gestational diabetes mellitus, a target group in general practice for preventive interventions: a populationbased cohort study," PLoS Medicine, vol. 15, no. 1, article e1002488, 2018.

[22] E. P. Gunderson, S. R. Hurston, X. Ning et al., "Lactation and progression to type 2 diabetes mellitus after gestational diabetes mellitus," Annals of Internal Medicine, vol. 163, no. 12, pp. 889-898, 2015.

[23] R. K. Peters, A. Xiang, S. L. Kjos, and T. A. Buchanan, "Longterm diabetogenic effect of single pregnancy in women with previous gestational diabetes mellitus," The Lancet, vol. 347, no. 8996, pp. 227-230, 1996.

[24] O. A. Henry and N. A. Beischer, "11 Long-term implications of gestational diabetes for the mother," Bailliere's Clinical Obstetrics and Gynaecology, vol. 5, no. 2, pp. 461-483, 1991.

[25] A. G. Ziegler, M. Wallner, I. Kaiser et al., "Long-term protective effect of lactation on the development of type 2 diabetes in women with recent gestational diabetes mellitus," Diabetes, vol. 61, no. 12, pp. 3167-3171, 2012.

[26] N. H. Cho, C. H. Ahn, J. H. Moon et al., "Metabolic syndrome independently predicts future diabetes in women with a history of gestational diabetes mellitus," Medicine, vol. 95, no. 35, article e4582, 2016. 
[27] W. Bao, D. K. Tobias, K. Bowers et al., "Physical activity and sedentary behaviors associated with risk of progression from gestational diabetes mellitus to type 2 diabetes mellitus," JAMA Internal Medicine, vol. 174, no. 7, pp. 10471055, 2014.

[28] H. Hakkarainen, H. Huopio, H. Cederberg, M. Pääkkönen, R. Voutilainen, and S. Heinonen, "Post-challenge glycemia during pregnancy as a marker of future risk of type 2 diabetes: a prospective cohort study," Gynecological Endocrinology, vol. 31, no. 7, pp. 573-577, 2015.

[29] S. H. Kwak, S. H. Choi, K. Kim et al., "Prediction of type 2 diabetes in women with a history of gestational diabetes using a genetic risk score," Diabetologia, vol. 56, no. 12, pp. 25562563, 2013.

[30] G. J. Cho, J. Heon Park, H. Lee, S. Yoo, S. Shin, and M. Oh, "Prepregnancy factors as determinants of the development of diabetes mellitus after first pregnancy," The Journal of Clinical Endocrinology \& Metabolism, vol. 101, no. 7, pp. 2923-2930, 2016.

[31] S. S. Casagrande, B. Linder, and C. C. Cowie, "Prevalence of gestational diabetes and subsequent type 2 diabetes among U.S. women," Diabetes Research and Clinical Practice, vol. 141, pp. 200-208, 2018.

[32] H. Lee, H. C. Jang, H. K. Park, B. E. Metzger, and N. H. Cho, "Prevalence of type 2 diabetes among women with a previous history of gestational diabetes mellitus," Diabetes Research and Clinical Practice, vol. 81, no. 1, pp. 124-129, 2008.

[33] Y. Wang, L. Chen, R. Horswell et al., "Racial differences in the association between gestational diabetes mellitus and risk of type 2 diabetes," Journal of Women's Health, vol. 21, no. 6, pp. 628-633, 2012.

[34] Y. Kugishima, I. Yasuhi, H. Yamashita et al., "Risk factors associated with the development of postpartum diabetes in Japanese women with gestational diabetes," BMC Pregnancy and Childbirth, vol. 18, no. 1, p. 19, 2018.

[35] R. Retnakaran and B. R. Shah, "Role of type 2 diabetes in determining retinal, renal, and cardiovascular outcomes in women with previous gestational diabetes mellitus," Diabetes Care, vol. 40, no. 1, pp. 101-108, 2017.

[36] E. Anderberg, K. S. Carlsson, and K. Berntorp, "Use of healthcare resources after gestational diabetes mellitus: a longitudinal case-control analysis," Scandinavian Journal of Public Health, vol. 40, no. 4, pp. 385-390, 2012.

[37] D. R. Coustan, M. W. Carpenter, P. S. O'Sullivan, and S. R. Carr, "Gestational diabetes: predictors of subsequent disordered glucose metabolism," American Journal of Obstetrics and Gynecology, vol. 168, no. 4, pp. 1139-1144, 1993.

[38] International Association of Diabetes and Pregnancy Study Groups Consensus Panel, "International association of diabetes and pregnancy study groups recommendations on the diagnosis and classification of hyperglycemia in pregnancy," Diabetes Care, vol. 33, no. 3, pp. 676-682, 2010.

[39] American Diabetes Association, "Diagnosis and classification of diabetes mellitus," Diabetes Care, vol. 37, Supplement 1, pp. S81-S90, 2014.

[40] WHO, Definition, diagnosis and classification of diabetes mellitus and its complications. Report of a WHO consultation, part 1: diagnosis and classification of diabetes mellitus, World Health Organisation, Geneva, 1999.

[41] The Committee on Nutrition and Metabolism of the Japan Society of Obstetrics and Gynaecology, "The committee report," Acta Obstetrica et Gynaecologica Japonica, vol. 36, pp. 2055-2058, 1984.

[42] J. E. Hux, F. Ivis, V. Flintoft, and A. Bica, "Diabetes in Ontario: determination of prevalence and incidence using a validated administrative data algorithm," Diabetes Care, vol. 25, no. 3, pp. 512-516, 2002.

[43] B. E. Metzger, "Summary and recommendations of the Third International Workshop-Conference on Gestational Diabetes Mellitus,” Diabetes, vol. 40, Supplement 2, pp. 197-201, 1991.

[44] T. Lind and P. R. Phillips, "Influence of pregnancy on the 75-g OGTT: a prospective multicenter Study," Diabetes, vol. 40, Supplement 2, pp. 8-13, 1991.

[45] C. G. Solomon, W. C. Willett, V. J. Carey et al., "A prospective study of pregravid determinants of gestational diabetes mellitus," JAMA, vol. 278, no. 13, pp. 1078-1083, 1997.

[46] National Diabetes Data Group, "Classification and diagnosis of diabetes mellitus and other categories of glucose intolerance," Diabetes, vol. 28, no. 12, pp. 1039-1057, 1979.

[47] K. G. M. M. Alberti and P. Z. Zimmet, "Definition, diagnosis and classification of diabetes mellitus and its complications. Part 1: diagnosis and classification of diabetes mellitus provisional report of a WHO consultation," Diabetic Medicine, vol. 15 , no. 7, pp. 539-553, 1998.

[48] L. Hoffman, C. Nolan, J. D. Wilson, J. J. N. Oats, and D. Simmons, "Gestational diabetes mellitus - management guidelines. The Australasian Diabetes in Pregnancy Society," Medical Journal of Australia, vol. 169, no. 2, pp. 93-97, 1998.

[49] American Diabetes Association, "Second WorkshopConference on Gestational Diabetes: summary and recommendations," Diabetes Care, vol. 34, Supplement 2, pp. 123126, 1985.

[50] D. A. Abell and N. A. Beischer, "The effects of hypoglycaemia and hyperglycaemia on pregnancy outcome," The Australian \& New Zealand Journal of Obstetrics \& Gynaecology, vol. 16, no. 2, pp. 75-81, 1976.

[51] Anonymous, "Diabetes mellitus. Report of a WHO Study Group," World Health Organ Tech Rep Ser, vol. 727, pp. 1113, 1985.

[52] T. E. Hopmans, C. van Houten, A. Kasius et al., "Increased risk of type II diabetes mellitus and cardiovascular disease after gestational diabetes mellitus: a systematic review," Ned Tijdschr Geneeskd, vol. 159, article A8043, 2015.

[53] C. Kim, K. M. Newton, and R. H. Knopp, "Gestational diabetes and the incidence of type 2 diabetes: a systematic review," Diabetes Care, vol. 25, no. 10, pp. 1862-1868, 2002.

[54] C. Song, Y. Lyu, C. Li et al., "Long-term risk of diabetes in women at varying durations after gestational diabetes: a systematic review and meta-analysis with more than 2 million women," Obesity Reviews, vol. 19, no. 3, pp. 421-429, 2018.

[55] G. Rayanagoudar, A. A. Hashi, J. Zamora, K. S. Khan, G. A. Hitman, and S. Thangaratinam, "Quantification of the type 2 diabetes risk in women with gestational diabetes: a systematic review and meta-analysis of 95, 750 women," Diabetologia, vol. 59, no. 7, pp. 1403-1411, 2016.

[56] International Diabetes Federation, IDF Diabetes Atlas, Diabetes Atlas, 8th edition, 2017.

[57] S. Behboudi-Gandevani, M. Amiri, Y. R. Bidhendi, and T. F. Ramezani, "The impact of diagnostic criteria for gestational diabetes on its prevalence: a systematic review and meta-analysis," Diabetology \& Metabolic Syndrome, vol. 11, no. 1, p. 11, 2019. 
[58] A. P. S. Kong, G. Xu, N. Brown, W. Y. So, R. C. W. Ma, and J. C. N. Chan, "Diabetes and its comorbidities-where East meets West," Nature Reviews Endocrinology, vol. 9, no. 9, pp. 537-547, 2013.

[59] WHO Expert Consultation, "Appropriate body-mass index for Asian populations and its implications for policy and intervention strategies," The Lancet, vol. 363, no. 9403, pp. 157-163, 2004.

[60] S. A. Lear, K. H. Humphries, S. Kohli, A. Chockalingam, J. J. Frohlich, and C. L. Birmingham, "Visceral adipose tissue accumulation differs according to ethnic background: results of the Multicultural Community Health Assessment Trial (MCHAT)," The American Journal of Clinical Nutrition, vol. 86, no. 2, pp. 353-359, 2007.

[61] The HAPO Study Cooperative Research Group, "Hyperglycemia and adverse pregnancy outcomes," The New England Journal of Medicine, vol. 358, no. 19, pp. 1991-2002, 2008.

[62] A. Duran, S. Sáenz, M. J. Torrejón et al., "Introduction of IADPSG criteria for the screening and diagnosis of gestational diabetes mellitus results in improved pregnancy outcomes at a lower cost in a large cohort of pregnant women: the St. Carlos Gestational Diabetes Study," Diabetes Care, vol. 37, no. 9, pp. 2442-2450, 2014.

[63] E. Noctor, C. Crowe, L. A. Carmody et al., “Abnormal glucose tolerance post-gestational diabetes mellitus as defined by the International Association of Diabetes and Pregnancy Study Groups criteria," European Journal of Endocrinology, vol. 175, no. 4, pp. 287-297, 2016. 